Service social

\title{
L'acte clinique et le geste technique dans la reconquête de l'identité professionnelle du travail social
}

\section{Belhassen Redjeb}

Volume 40, numéro 2, 1991

Formation et évolution de la pratique en travail social

URI : https://id.erudit.org/iderudit/706530ar

DOI : https://doi.org/10.7202/706530ar

Aller au sommaire du numéro

\section{Éditeur(s)}

École de service social de l'Université Laval

ISSN

1708-1734 (numérique)

Découvrir la revue

Citer cet article

Redjeb, B. (1991). L'acte clinique et le geste technique dans la reconquête de l'identité professionnelle du travail social. Service social, 40(2), 105-115.

https://doi.org/10.7202/706530ar
Résumé de l'article

Après avoir constaté et souvent contesté l'atomisation de sa pratique professionnelle par et dans les lieux de travail fortement organisés, l'intervenant social s'engage de plus en plus dans une démarche de réappropriation de sa pratique par la reconstitution et la revendication de son acte fondateur, l'acte clinique. Or, celui-ci est irréductible, croit l'auteur, aux gestes techniques posés quotidiennement par l'intervenant social : les constituantes, de même que les conditions de possibilité et de permanence du premier, diffèrent ostensiblement de celles propres aux seconds.

Dans cet article, l'auteur tente d'abord d'établir ce qui revient à l'un et à l'autre, sachant bien que c'est seulement au terme de cet exercice qu'il pourra situer l'intervention sociale d'aujourd'hui et localiser ce qui lui fait défaut pour retrouver son sens d'acte clinique. Cet examen le conduira, pour finir, à remettre en perspective les conditions de restitution de l'acte clinique de l'intervention sociale. 
Belhassen Redjeb, professeur au Département de techniques en travail social, Cégep de Lévis-Lauzon, et chargé de cours à l'École de service social de I'Université Laval.

\section{L'acte clinique et le geste technique dans la reconquête de l'identité professionnelle du travail social'}

Belhassen Redjeb

Après avoir constaté et souvent contesté l'atomisation de sa pratique professionnelle par et dans les lieux de travail fortement organisés, l'intervenant social s'engage de plus en plus dans une démarche de réappropriation de sa pratique par la reconstitution et la revendication de son acte fondateur, I'acte clinique ${ }^{2}$. Or, celui-ci est irréductible, croyons-nous, aux gestes techniques posés quotidiennement par l'intervenant social au sein des organisations modernes d'État : les constituantes, de même que les conditions de possibilité et de permanence du premier, diffèrent ostensiblement de celles propres aux seconds.

Dans cet article, nous nous proposons d'abord d'établir ce qui revient à l'un et à l'autre, sachant que c'est seulement au terme de cet exercice que nous pourrons situer l'intervention sociale d'aujourd'hui et tenter de localiser ce qui lui fait défaut pour se constituer en acte clinique. Cet examen nous amènera, dans un deuxième temps, à mettre de l'avant certaines conditions qui nous paraissent pertinentes pour restituer les exigences relatives à l'acte clinique de l'intervention sociale, acte autour duquel s'édifie l'identité professionnelle.

Nul doute qu'un des procédés les plus féconds pouvant conduire à une réflexion sérieuse sur le travail social d'aujourd'hui, c'est celui qui consiste à scruter ce qui est considéré comme sa base, à savoir l'acte clinique. Cet angle d'attaque, sans être le seul, nous apparaît d'autant plus approprié que les lieux fortement organisés dans lesquels il s'exerce, comme les organismes du réseau public des services sociaux, l'ont morcelé au point de le réduire à une série de gestes techniques rarement exécutés par la même personne. Cette atomisation de l'acte clinique du travail social a été constatée, controversée et 
contestée $^{3}$. Pour ses tenants, cette atomisation est immanente et irréversible compte tenu des vertus prééminentes de l'organisation rationnelle centrées sur la productivité et le contrôle de la productivité, eu égard au critère de l'efficacité. Pour les opposants, l'atomisation est une "mutilation » de l'acte clinique et, par ricochet, de l'identité professionnelle de celui qui en assume l'exercice. Elle a pour effet de dénaturer le sens de l'acte clinique et de le rendre vulnérable à d'autres structurants tels que les lois et les programmes.

Ces éléments de bilan de l'itinéraire de l'acte clinique au sein des organisations modernes d'État sont du reste connus de la presque totalité des intervenants sociaux concernés par l'acte en question. Les deux positions qui se dégagent de ce bilan sommaire sont souvent qualifiées comme suit : celle qui prône l'atomisation, on l'appelle la position gestionnaire; elle confond l'acte clinique et le geste technique en érigeant ce dernier au rang du premier. L'autre, la position professionnelle, est celle qui affirme que l'acte clinique ne saurait être réduit aux gestes techniques en ce sens qu'il est une totalité et, de ce fait, non réductible à la somme de ses parties et encore moins à une de ses parties, en l'occurrence le geste technique.

Afin de mieux situer ces deux positions et leur impact sur la représentation de l'identité professionnelle, examinons de près les constituantes paradigmatiques de l'acte clinique et du geste technique ainsi que les conditions de possibilité et de permanence de l'un et de l'autre.

\section{Constituantes paradigmatiques de l'acte clinique et du geste technique}

Le mot clinique est dérivé du grec klinè qui veut dire « lit ». Dans la pratique médicale, l'activité clinique consistait initialement à observer le malade couché afin de connaître son état. La psychanalyse a retenu cette posture pour son sujet et la psychologie clinique a emprunté l'essentiel du procédé clinique tout comme plus tard le travail social, par la méthode du casework, s'est édifié autour de la démarche clinique. Ce rappel est important si l'on veut retracer les composantes de l'acte clinique, composantes qui sont sensiblement les mêmes dans la pratique médicale, dans la pratique psychologique (I'expérimentation en moins) ainsi que dans la pratique idéale du travail social. Bien entendu, le contenu et l'objet de l'acte clinique de chacune de ces pratiques sont postulés distincts.

- La première composante de l'acte clinique est l'observation : "le regard clinique ", comme l'appelle Foucault, " a cette paradoxale 
propriété d'entendre un langage au moment où il perçoit un spectacle $^{4}$ "; riche énoncé voulant signifier que l'observation clinique doit sa pureté au silence et à l'absence d'interférences, afin " que les choses vues puissent être entendues, et entendues par le seul fait qu'elles soient vues ${ }^{5}$ ». C'est aussi voir et savoir en même temps, puisque l'on s'instruit de ce qu'on observe.

- La seconde composante de l'acte clinique est celle de l'analyse compréhensive ou, si l'on veut, de la compréhension par l'analyse. $C^{\prime}$ est à ce moment que le regard clinique, avec le soutien de la logique et de la connaissance antérieure de l'objet d'observation, se détache, se découple de l'immédiat visible, se décentre et laisse la place au raisonnement et à la reconstruction intelligible des éléments observés.

- La troisième composante de l'acte clinique, c'est le diagnostic qui consiste à porter, en vue d'une intervention (dans le cas du travail social), un jugement hypothétique sur la situation, jugement éclairé par, d'une part, les fruits de l'observation et de l'analyse compréhensive et, d'autre part, par des principes d'ordre éthique. Ces principes ne doivent pas être confondus avec les règles du code de déontologie en ce sens que les principes d'ordre éthique sont des fondements moraux au jugement (par exemple : le bien, le juste, le sincère et leurs équivalents), tandis que les règles déontologiques sont des prescriptions de l'ordre de la procédure applicables à l'ensemble de l'acte clinique (par exemple : la confidentialité). En outre, le principe d'ordre éthique inspire la question de savoir ce qu'on doit faire dans une situation donnée, alors que la règle déontologique indique comment on doit faire dans une situation donnée ${ }^{6}$.

- La quatrième et dernière composante de l'acte clinique est celle de l'intervention sur la situation, intervention conçue à partir du jugement (diagnostic) qui la rend impérative. En plus de mobiliser certains savoirs, l'intervention s'effectue dans un contexte qui l'informe.

Ces constituantes de l'acte clinique en travail social étant brièvement esquissées, il importe maintenant de poser les conditions de possibilité et de permanence de cet acte clinique, conditions congruentes, il va sans dire, avec la nature de ses constituantes.

- La première condition a trait à la distance que se doit de garder l'acteur chargé d'accomplir l'acte clinique par rapport aux conditionnements et arrangements sociaux formalisés dans des organisations et provenant d'autres sources que celles qui fondent l'acte clinique. Cette condition d'autonomie peut être modulée selon les exigences de I'acte clinique, c'est-à-dire celles de l'observation, de l'analyse, du jugement à porter et de l'intervention à effectuer. Toutefois, une pareille distance ne doit pas être interprétée en tant qu'indifférence à 
l'égard de ces conditionnements, mais plutôt comme une "posture " de l'esprit qui lutte contre les interprétations toutes faites de l'objet clinique.

- La seconde condition de possibilité et de permanence de l'acte clinique est la maîtrise de connaissances pertinentes et reproductibles (par la formation) et, bien entendu, perfectibles (par la recherche). En outre, les emprunts à d'autres disciplines au profit de celle du travail social, en l'occurrence, permettent cette reproductibilité et cette perfectibilité à condition qu'ils subissent des traitements propres à la discipline du travail social; sans quoi on ne fait que " contrefaire » les autres disciplines, mettant ainsi en péril la légitimité et la permanence de cette discipline du travail social et donc de son acte clinique en propre.

- La troisième et dernière condition de possibilité et de permanence de l'acte clinique, c'est son intégrité qu'il ne faut pas confondre avec son intégralité. L'acte clinique est une totalité, avons-nous posé au départ, irréductible donc à la somme de ses parties. C'est dire que ce n'est pas seulement les quatre composantes qui maintiennent l'intégrité de l'acte clinique, mais aussi et surtout les interactions entre celles-ci, interactions qui font sens, le sens de l'acte clinique. Dès lors, altérer l'intégrité de l'acte clinique, que ce soit par atomisation de ce dernier ou de ses composantes, revient à altérer le sens de l'acte clinique même, puisque les interactions qui fondent la totalité s'évanouissent dans les méandres de la division du travail, comme en fait foi la formule "A.E.O. " (accueil, évaluation, orientation) largement utilisée dans les établissements de services sociaux?

Les composantes de l'acte clinique, de même que ses conditions de possibilité et de permanence, étant brièvement exposées, il importe, dès l'instant, de faire le même exercice pour le geste technique :

- La première composante du geste technique est l'instrument qui lui permet de se matérialiser. Cet instrument est en même temps actualisé par le geste en question; par exemple, les techniques d'entrevue, qui sont des instruments, permettent de faire l'entrevue qui devient le geste technique; ce faisant, les techniques s'actualisent dans et par le geste.

- La deuxième composante du geste technique, c'est l'effort efficient. En principe, le geste technique ne peut être la cause que de l'effet pour lequel l'instrument est conçu ${ }^{8}$ (un effort efficient idéal est celui où le rapport entre ce qui est réalisé et les moyens mis en œuvre est égal à 1). Il s'ensuit que la personne qui fait le geste technique ne peut définir son intention d'action qu'à partir ou en fonction de l'ef- 
ficience de la technique, à moins que le geste technique ne soit détourné du dessein que lui réserve l'instrument qui le rend possible.

Ajoutons qu'un geste technique, pour être fait, appelle un technicien formé en conséquence. Une division technique du travail permet donc au geste technique d'actualiser son efficience selon un ordre donnés; d'où la nécessité de l'encadrement sans lequel le geste technique devient absurde. À ces deux conditions de possibilité et de permanence du geste technique s'ajoute la nécessaire dépendance du geste technique, et donc du technicien, à un ensemble structuré qui endigue le sens du geste technique et le dote, en échange, d'une légitimité.

L'acte clinique et le geste technique ont des constituantes paradigmatiques distinctes : I'un ne peut se confondre avec l'autre sous peine de prendre la totalité pour la partie ou, ce qui revient au même, l'inverse. Ces deux formes quasi archétypales de l'action permettent, au surplus, de classer les pratiques du travail social selon qu'elles s'apparentent à l'acte clinique ou au geste technique. Notre point de vue, à cet égard, est le suivant :

De façon générale (il s'agit bien d'une tendance), le type de pratique et plus particulièrement d'intervention en vigueur au sein des organisations modernes d'État, organisations fortement différenciées et organisées, se rapproche ostensiblement davantage du geste technique que de l'acte clinique. Cependant, dans les réseaux parallèles à celui de l'État, notamment celui de la pratique privée et celui des pratiques dites alternatives, l'intervention prend davantage la forme de I'acte clinique que celle du geste technique.

Par ailleurs, on persiste à donner la formation à l'intervention sur le modèle de l'acte clinique bien que, dans les stages de formation pratique, cette observation soit moins évidente. Là encore, la situation peut varier selon les lieux de stages, les représentations du stagiaire, du superviseur et du consultant de la pratique du travail social.

À vrai dire, l'acte clinique n'exclut pas le geste technique en ce sens que le premier comprend le second. Le problème se pose à partir du moment où le geste technique s'impose parfois subrepticement et $d^{\prime}$ 'autres fois ostensiblement comme un acte clinique alors que, dans les faits, il n'en est qu'un composant. C'est comme si l'on disait à un médecin que, désormais, son acte clinique consiste soit à manipuler un stéthoscope, soit à prescrire un médicament ou à assurer un suivi, mais pas les trois gestes intégrés dans un ensemble. C'est comme si l'on réduisait l'acte clinique du psychologue à des entrevues d'exploration ou d'évaluation, alors que d'autres se chargeraient de faire le reste, soit l'expérimentation ou la psychothérapie proprement dite. $\mathrm{Si}$ ce n'est pas encore le cas pour ces deux corps professionnels, pour- 
quoi le serait-ce dans le cas du travail social ? Car tout le monde sait que l'acte clinique en ce qui concerne le travail social exercé dans les organisations modernes d'État connaît un éclatement sans précédent. Non seulement les lois et les programmes ordonnent-ils (et le terme n'est pas trop fort) aux intervenants sociaux quoi faire et comment le faire, mais en plus ils substituent à l'analyse compréhensive et au jugement en vue de l'action du " prêt-à-penser » qui laisse peu de place à l'autonomie de la pensée et du jugement.

Comment dès lors reconquérir l'acte clinique, noyau de l'identité professionnelle des intervenants sociaux, dans un contexte fortement organisé où le geste technique prévaut sur d'autres formes d'intervention sociale ? Il y $a$, nous semble-t-il, matière à esquisser un itinéraire à suivre dans cette reconquête de l'identité professionnelle au travail. Pour ce faire, commençons par annoncer certaines conditions de départ sans lesquelles persisteraient des ambiguïtés quant à la nature de la démarche.

\section{Les conditions de l'identité professionnelle}

1. La reconquête de l'identité professionnelle exige, d'abord, que les intervenants sociaux se dotent d'une représentation claire et précise de l'acte clinique, en ce sens que cette représentation soit fondée historiquement et non pas due uniquement à un exercice mental d'ordre cosmétique.

2. L'encadrement professionnel est nettement distinct de l'encadrement administratif; la fusion des deux fonctions telle que nous l'observons dans certains établissements ne peut garantir l'autonomie de l'acte clinique et de son encadrement strictement professionnel; en clair, les impératifs de la gestion ne sont pas les mêmes que ceux de la profession et la gestion des services sociaux ne doit pas se confondre avec la supervision de l'acte en travail social, l'acte clinique.

3. La gestion des établissements des services sociaux a certainement sa place dans le service offert à la clientèle, mais ne devrait sûrement pas avoir la prédominance dans l'encadrement professionnel, comme c'est actuellement le cas. Cette situation est d'ailleurs déplorée par les membres de la Commission Rochon quand ils affirment que les " praticiens relèvent d'un gestionnaire sur le plan administratif et professionnel » et que l'encadrement professionnel proprement dit fait carrément défaut ${ }^{10}$.

Avant de procéder à la mise en perspective de ces trois conditions, il nous paraît important de spécifier qu'elles sont interactives au sens où le sort de l'une dépend du sort des autres. Par exemple, on aura beau se doter d'une représentation claire et précise de l'acte clinique 
en travail social, cet exercice restera futile si la gestion continue à dominer l'acte professionnel en faisant prévaloir ses prérogatives dans la définition non seulement du service, mais aussi de l'intervention sociale ou de l'acte clinique en l'englobant dans son objet. De plus, une stratégie appropriée se doit d'accompagner l'instauration de tout itinéraire qui se donne ces trois conditions de départ. D'abord, s'assurer de lieux de réflexion et de concertation entre pairs et aussi de lieux de dialogue avec les pairs de souche qui sont devenus gestionnaires des services sociaux. Ensuite, il nous semble important de se doter de ce que nous appelons une éthique de discussion qui dépasse la simple morale procédurière (les règles de procédure), le fair play ou la discussion complaisante.

Une mise en perspective des trois conditions annoncées ci-dessus pour rendre plausible l'idée de la reconquête de l'identité professionnelle commande le développement de chacune de façon opérationnelle.

1. Pour ce qui est de l'acte clinique, sa représentation claire, précise et fondée historiquement requiert qu'on libère l'expression " acte clinique » du sens péjoratif qui l'accable et que certaines idéologies de circonstance, (y compris même l'idéologie corporatiste) surtout au Québec, lui ont attribué. À cet égard, les constituantes de l'acte clinique, de même que ses conditions de possibilité et de permanence telles que nous les avons vues ci-dessus, constituent, à notre avis, une trame sur laquelle il est possible d'articuler les éléments de l'acte clinique puisés dans l'histoire du travail social et dans l'expérience du présent.

La représentation postulée de l'acte clinique procède donc du sens que l'on donne à l'acte clinique. La restitution du véritable sens de l'acte clinique en travail social exige une reconstruction historique de son contenu, tâche d'autant plus pénible à effectuer que la communauté des intervenants sociaux (formateurs compris) a tendance à céder facilement devant l'exigence pragmatique de l'ici et maintenant. De sorte que les éléments du savoir en travail social s'accumulent sans toutefois être dépouillés de leurs contenus vétustes ou circonstanciels. Une histoire de la connaissance en travail social permettrait de reconstruire le sens de l'acte clinique à condition, bien entendu, que cette entreprise soit menée selon des exigences universelles de validité. Car, pour dégager les constituantes de l'acte clinique des humeurs idéologiques affichées par les contextes sociaux de la pratique du travail social, il faudrait bien sûr une démarche historicoscientifique qui ne tolère aucun a priori sans l'avoir sérieusement mis en question. Pour ce faire, on aurait intérêt à s'inspirer de la réflexion de Thomas S. Khun sur le rôle de I'histoire dans le développement de la science ${ }^{11}$. 
En somme, l'acte clinique ne doit pas être idéologiquement chargé, contrairement à ce qu'il a été et à ce qu'il est encore, que ce soit de façon dissimulée ou explicite. Cependant, il doit être éthiquement fondé. Là encore, l'éthique a subi un réductionnisme, notamment chez les intervenants sociaux, par suite d'usages de circonstance qui l'ont confondue avec la déontologie ${ }^{12}$ ou la morale juridique ${ }^{13}$. L'éthique est une pratique de questionnement qui examine la morale sous toutes ses formes, questionnement qui permet de réouvrir la question du sens et de ce qui est donné comme allant de soi. En tant que discipline d'action régulatrice, le travail social ne peut échapper au questionnement éthique sous peine de voir la normativité de son acte clinique interpellée par les critiques sociaux. Son positionnement normatif au moment de la formulation d'un jugement d'action doit être éthiquement fondé, c'est-à-dire soumis à une discussion rigoureuse menée selon des exigences de validité. La réalisation d'une telle démarche serait de nature à doter l'acte clinique d'un sens, d'une pertinence et d'une légitimité autres que ceux octroyés par d'autres pratiques, notamment les pratiques administratives et légales. Corollairement, cette démarche constitue un pas de plus vers l'autonomie et l'identité professionnelles.

2. Pour ce qui est de l'encadrement professionnel, qui doit être nettement distinct de l'encadrement administratif, la ligne de conduite est celle qui reconnaît qu'il y a des exigences propres à l'acte clinique et qui ne sont pas de la même nature que celles qui sont intrinsèques à la pratique gestionnaire. Cette proposition est d'autant mieux fondée que la première condition relative à la restitution du sens de l'acte clinique est remplie. Dès lors que l'on établit la nécessité de ne pas substituer à l'encadrement de l'acte clinique l'encadrement administratif, toute interférence des deux s'estompe. Cette distinction est elle-même d'ailleurs bien établie dans des professions comme la médecine; elle est plutôt problématique dans ce que A. Etzioni appelle les "semi-professions ${ }^{14}$ " dont ferait partie le service social.

3. La place de la gestion dans le service à la clientèle et celle de l'acte clinique dans le même service ne devraient pas avoir à se concurrencer et encore moins à se tourner le dos; il y a, nous semble-t-il, place à la convergence. Encore une fois, la définition de l'acte clinique faite de façon claire et précise est la condition de départ pour engager un dialogue sur la place qui lui revient et celle qui revient à la gestion dans le service à la clientèle. On le sait, la qualité du dialogue est corrélative du degré de reconnaissance effective des parties qui y prennent part. Or, cette condition n'est pas tout à fait remplie quand on examine les rapports entre les différents groupes d'acteurs impliqués directement ou indirectement dans la production de l'acte clinique $^{15}$. Le dialogue entre formateurs, intervenants, agents de I'en- 
cadrement professionnel et agents de l'encadrement administratif est rare parce que peu institué; et quand il a lieu, l'ambiguïté des enjeux est telle qu'il est souvent difficile de découpler les intérêts de l'argumentation. Sans compter que d'autres conditions, qui rendraient le dialogue possible, ne sont pas réunies de façon probante : on pense notamment à une infrastructure qui permettrait d'institutionnaliser les échanges et les discussions et, surtout, ce qui est au centre de la présente analyse, une réappropriation réflexive par les intervenants sociaux de l'ensemble de l'acte clinique de l'intervention sociale, de même que des dimensions administratives de celui-ci. Car, dès lors que l'intervenant social indexera le facteur administratif au donné clinique de son intervention (et non l'inverse comme on est porté à le faire aujourd'hui), il y aura, conjecturons-nous, une meilleure intégration du premier au second. C'est une condition d'efficience que le dialogue aura à remplir. Car, cette condition remplie, il sera possible d'apprécier la part de l'administratif dans la portée de l'acte clinique; à défaut de quoi, il faudra plutôt se résigner à scruter ce qui reste de clinique dans le geste technique.

\section{Conclusion}

Nous ne prétendons pas que la présente réflexion à propos de l'acte clinique et du geste technique dans la reconquête de l'identité professionnelle du travail social soit exhaustive. Tout au plus, considérons-nous avoir circonscrit le noyau de cette réflexion. Les exigences de la rigueur plaident en faveur d'un examen plus détaillé de ce que nous avons esquissé, examen difficile à contenir dans l'espace alloué dans le présent contexte. Ainsi, bien que la distinction entre l'acte clinique et le geste technique soit introduite, elle n'est établie que de façon élémentaire pour convenir de la différence entre les deux. Cette différence fonde et autorise la restitution de l'acte clinique en travail social aussi bien dans ses composantes que dans le contenu de chacune de ces composantes. Cette restitution annoncée reste à faire et nous avons mis de l'avant certaines conditions de faisabilité dont celle ayant trait à la démarche historico-scientifique qu'il importe d'adopter pour ce faire. Une telle démarche devrait se solder par l'édification $d^{\prime}$ une représentation recevable de l'acte clinique en travail social. Elle permettrait, de surcroît, de poser la nécessité de différencier l'encadrement professionnel et l'encadrement administratif. Cette seconde condition est redevable à la première tout en la renforçant. Leur impact cumulatif faciliterait la satisfaction de la troisième condition ayant trait à la localisation du geste technique, soit l'apport administratif, dans l'acte clinique. S'engager à remplir les conditions de restitution de 
l'acte clinique, c'est en même temps œuvrer dans le sens de l'identité professionnelle. En effet, la corrélation entre les deux niveaux d'une même réalité, le travail social, est étroite en ce que l'évocation de l'un ne peut se faire sans l'implication de l'autre.

\section{Notes}

${ }^{1}$ Cet article est tiré du texte d'une conférence donnée au Centre de services sociaux du Centre du Québec, Trois-Rivières, dans le cadre d'une journée d'étude organisée par le Conseil consultatif du personnel clinique et portant sur la reconquête de l'identité professionnelle, le 16 mars 1990.

${ }^{2}$ Mentionnons la réflexion toute récente de Sylvie Dagenais, "La qualité totale : un idéal pour les services sociaux ? ", dans Intervention, revue de la Corporation professionnelle des travailleurs sociaux du Québec, $\mathrm{n}^{\circ} 87$, janvier 1991, p. 80-85.

${ }^{3}$ Marcelle Laforest et Belhassen Redjeb, Le service social dans les Centres de services sociaux au Québec: une double réalité, rapport de recherche, Laboratoire de recherche de l'École de service social, Université Laval, Québec, 1983, p. 103-141.

${ }^{4}$ Michel Foucault, Naissance de la clinique, Paris, Presses universitaires de France, 1963, p. 108.

${ }^{5}$ Ibid., p. 115.

${ }^{6}$ Pierre Fortin, "L'éthique et la déontologie : un débat ouvert dans l'éthique professionnelle ", dans Cahiers de recherche éthique 13, Montréal, La Corporation des Éditions Fidès, 1989, p. 65-82.

${ }^{7}$ L'accueil, l'évaluation et l'orientation sont trois gestes qui se présentent en principe comme des préalables aux gestes propres à l'intervention et au suivi que commande l'intervention.

${ }^{8}$ Jacques Ellul, Le système technicien, Paris, Calmann-Lévy, 1977, p. 31-42.

${ }^{9}$ Ibid., p. 32.

${ }^{10}$ Gouvernement du Québec, "Les professionnels et leurs pratiques ", dans Rapport de la Commission d'enquête sur les services de santé et les services sociaux, 1988, p. 292.

11 Thomas S. Khun, La structure de la révolution scientifique, Paris, Flammarion, 1972 , p. 17-24.

${ }^{12}$ Les corporations professionnelles ont tendance à utiliser indistinctement l'expression " code d'éthique " et " étude de déontologie ".

${ }^{13}$ Joseph Pestiau, "Droit, éthique, économie», dans Éthique et droits fondamentaux, publié sous la direction de Guy Lafrance, Les Presses de I'Université d'Ottawa, 1989, p. 195-200.

${ }^{14}$ « ...les superviseurs de travail social ont tendance à s'orienter davantage vers I'organisation et moins vers le client même dans le travail quotidien, et ils voient eux-mêmes peu de cas, pour ne pas dire aucun, une fois qu'ils grimpent dans la hiérarchie ", Amitai Etzioni, Les organisations modernes, Gembloux, Duculot, 1971, p. 161. 
15 "Dans le domaine social, ce sont les établissements qui définissent les clientèles à desservir et les services à offrir. Ceux-ci décident de l'équilibre à maintenir entre leur mission, les demandes de services, les ressources et les moyens à utiliser. Aux orientations des établissements, s'ajoutent les priorités gouvernementales et l'application des différentes lois, telles que la Loi sur la protection de la jeunesse, qui réduisent la marge de manœuvre des praticiens sociaux ", Gouvernement du Québec, Rapport de la Commission d'enquête sur les services de santé et les services sociaux, 1988, p. 292. 